\title{
ESTUDO DA RELAÇÃO ENTRE PRECIPITAÇÃO E DESLIZAMENTOS NO MUNICÍPIO DE PETRÓPOLIS - RJ
}

Giselle Petrungaro Torres giselle.petrungaro@gmail.com Universidade Federal do Rio de Janeiro

Luiz Felipe Rodrigues do

\section{Carmo}

I.docarmo.meteoroufrj@gmail. com

Universidade Federal do Rio de Janeiro

Ana Cristina Pinto de Almeida Palmeira

anactn@gmail.com

Universidade Federal do Rio de Janeiro

\section{RESUMO}

O município de Petrópolis, na Região Serrana do estado do Rio de Janeiro, por muitas vezes é atingido por desastres naturais, como deslizamentos de terras. Na maioria das vezes são desencadeados por eventos meteorológicos. Devido a essa problemática, foi realizado este estudo que tem como base a relação entre atmosfera e solo, para evidenciar esta relação citada e possibilitar traçar estratégias de prevenção e mitigação dos impactos de tais eventos. Foram utilizados dados de ocorrência de deslizamentos, classificados de acordo com o tipo, em conjunto com os registros de precipitação no município de Petrópolis em determinada série temporal e através de ferramentas gráficas computacionais, foram geradas correlações, análises temporais e espaciais. Os resultados preliminares mostraram a maior relação entre acumulados de chuva e determinadas classificações de deslizamentos. Um exemplo foi no caso das corridas de lama, no qual mostrou a relação destes eventos com as características do local da ocorrência.

Palavras-chave: Precipitação; Deslizamento; Petrópolis 


\section{INTRODUÇÃO}

Segundo o Instituto Brasileiro de Geografia e Estatística (IBGE, 2020), o município de Petrópolis, localizado na Região Serrana do estado do Rio de Janeiro, é o $16^{\circ}$ em área territorial do estado, sendo dividido em cinco distritos: Petrópolis, Cascatinha, Itaipava, Pedro do Rio e Posse. Conforme a Figura 1, o município tem como importante característica a diversidade espacial e da utilização do solo, sendo formado por áreas rurais e urbanas, com altitude média de 845 metros.

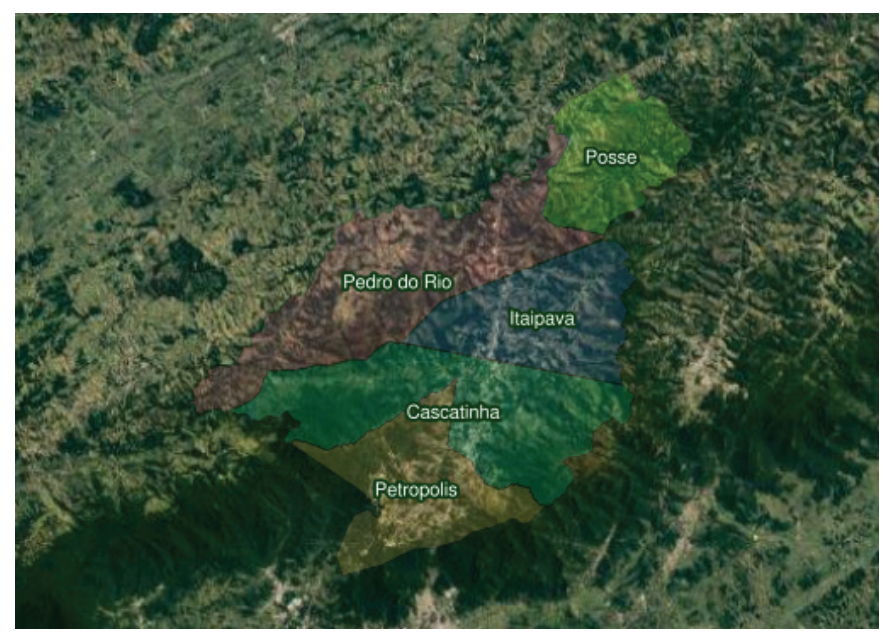

Figura 1. Distritos do Município de Petrópolis.

Fonte: Prefeitura Municipal de Petrópolis e Google.

O município de Petrópolis está localizado na Área de Proteção Ambiental (APA) da Região Serrana de Petrópolis, criada em 1982, tendo como bioma predominante a Mata Atlântica, segundo o Instituto Chico Mendes de Conservação da Biodiversidade (ICMBio, 2020). O intuito da criação desta APA foi a preservação dos remanescentes da Mata Atlântica, o uso sustentável dos recursos naturais, a conservação do seu conjunto cultural e paisagístico, e a melhoria da qualidade de vida na região. Contudo, com o passar dos anos e o desenvolvimento urbano do município, a vegetação nativa foi substituída por uma vegetação secundária ou foi desmatada para dar lugar a ocupações irregulares e desordenadas (Guerra et al., 2007), o que aumenta a vulnerabilidade da região.

O município de Petrópolis está compreendido, em sua maioria, na Região Hidrográfica do Piabanha (RH IV), e parcialmente na Região Hidrográfica da Baía de Guanabara ( $\mathrm{RH}$ V) (INEA, 2013), as quais são gerenciadas por comitês que têm como objetivo, entre outros, melhorar a qualidade das bacias hidrográficas e atuar ativamente em questões relativas à poluição e ao desmatamento naquela área (INEA, 2020).

O município de Petrópolis, que se localiza em uma região com expressiva declividade e relevo, é, muitas vezes, atingido por desastres naturais, como transbordamentos, enxurradas e deslizamentos - foco deste estudo. Como exemplo, o Rio Quitandinha é responsável pelo maior histórico de ocorrências de transbordamentos do estado, com rápido tempo de resposta às chuvas que ocorrem na área de contribuição da bacia (Carmo et al., 2018). A viabilidade destes eventos, somada à crescente ocupação urbana, por vezes desordenada, expõe a cidade a eventos que possuem grande potencial destrutivo, como já ocorrera diversas vezes. Em 2011, a Região Serrana do estado do Rio de Janeiro foi atingida por mais de 3.000 deslizamentos, resultando na morte de milhares de pessoas e gerando graves prejuízos à infraestrutura urbana e rural da região (Coelho Netto et al., 2013).

O evento geológico chamado deslizamento possui diferentes definições com pequenas diferenças entre si. Segundo Highland e Bobrowsky (2008), é compreendido como o movimento de descida do solo, rochas e de material orgânico, sob efeito da gravidade, e também a formação geológica resultante deste movimento. Estes autores afirmaram que os eventos podem ser classificados de acordo com a ocorrência, extensão, taxa de movimento, mecanismo de desencadeamento e efeitos. É possível também, dentro da classificação de cada evento, traçar alguma previsibilidade do mesmo e por conseguinte buscar medidas mitigadoras.

Para que seja classificado como um desastre natural, seja em casos de deslizamento ou transbordamento, é necessário que haja impacto na região em relação à população humana ou ao meio ambiente. Desastres podem ser bem entendidos se analisados como resultado da complexa interação entre um evento físico potencialmente prejudicial, como os citados anteriormente, e a vulnerabilidade de uma sociedade a ele exposta, segundo Licco (2013). Estes eventos podem ter diferentes graus de impacto e as pessoas atingidas, assim como a região, podem ter dificuldade de reestruturação que podem levar anos.

Por vezes, deslizamentos e transbordamentos ocorrem no mesmo período e até de forma simultânea, por estarem ligados a ocorrência de chuva na região, que é um grande mecanismo desencadeador dos desastres naturais. Com isso, é necessário que se conheça o potencial de ocorrência dos eventos extremos no local, considerando possíveis cenários quanto a condições atmosféricas. Ou seja, é necessário o estudo da região de modo a prever a reação dessas ocorrências frente às condições meteorológicas extremas.

Chuvas intensas atingem a Região Serrana, principalmente no verão, caracterizado como o período chuvoso. Nesta época do ano, foram registradas as maiores ocorrências de deslizamentos, principalmente nos últimos anos. Vale ressaltar que estes eventos são resultantes da combinação de fatores geológicos, geomorfológicos, hidrológicos e climáti- 
cos, além da ocupação desordenada das encostas, segundo Salles e Amaral (2013).

Segundo D'orsi (2011), na Região Sudeste do Brasil, os eventos de chuvas intensas com consequentes ocorrências de escorregamentos são frequentes e com o passar dos anos são registradas dezenas de novos episódios. Em geral, como estes processos impactam seriamente e negativamente na sociedade e no ambiente ao redor, são realizados diversos estudos através de diferentes metodologias ao redor do mundo, a fim de estabelecer e compreender melhor os índices pluviométricos capazes de desencadear estes eventos.

Desta forma, o objetivo deste trabalho é relacionar estatisticamente os eventos de deslizamentos com as ocorrências de chuvas no município de Petrópolis para que, a partir destas informações, os órgãos responsáveis pela gestão do município (Defesa Civil e a Prefeitura de Petrópolis) possam estar sempre em sincronia com profissionais de cada uma das áreas interessadas e, consequentemente, utilizar estas informações para traçar estratégias de prevenção, de orientação, de mitigação e de resiliência no caso da ocorrência de eventos.

\section{DADOS E MÉTODOS}

Para este estudo foram utilizados dados das ocorrências de eventos de deslizamentos no município de Petrópolis, compreendidos entre os anos de 1940 e 1990, obtidos pelo Instituto de Pesquisas Tecnológicas (IPT). O IPT contou com dados adquiridos de observações diretas, de relatos pessoais e registros em jornais e revistas publicados na época. Foram também utilizados os dados de deslizamentos compreendidos entre os anos 2000 e 2015, cedidos pelo Laboratório de Cartografia (GEOCART), do Departamento de Geografia da Universidade Federal do Rio de Janeiro, sendo abordado com diferente metodologia em relação aos dados do período anterior.
Os dados dos deslizamentos foram correlacionados com as precipitações da estação pluviométrica de Petrópolis do IPT - Posto 1, localizada no primeiro distrito do município. Juntamente com essas informações foram consideradas as contagens de vítimas associadas a cada evento registrado, de forma a relacionar o tipo de evento com o potencial de perigo que apresenta.

Os eventos dos deslizamentos registrados foram classificados previamente pelo GEOCART, em cinco categorias, segundo a metodologia do IPT: escorregamento (E), queda de blocos (QB), rolamento de blocos (RB), corrida de lama (CL) e diversos (D), como adaptado no Quadro 1.

A partir dos dados obtidos pelos institutos IPT e GEOCART (cada um em determinado período e de acordo com o volume de dados de cada período), foram realizadas análises distintas para cada conjunto de informações. Este agrupamento foi feito de forma a apresentar uma análise temporal dos dados referentes ao IPT, compreendidos entre os anos de 1940 e 1990, além de uma análise espacial referente aos dados do GEOCART, compreendidos entre os anos de 2000 e 2015.

Para elaborar a relação entre as ocorrências de eventos, a classificação quanto ao tipo de evento geológico e os acumulados de precipitação na estação, foi feito o processamento dos dados para que fossem excluídos dados espúrios de registro de chuva. O processamento de dados avaliou a ocorrência de erros sistemáticos no registro da precipitação no pluviômetro utilizado. Após analisados e confirmados como dados espúrios foram desconsiderados na análise realizada.

A análise dos dados foi feita através de ferramentas computacionais matemáticas e gráficas, sendo realizada por etapas. A ferramenta computacional matemática e gráfica utilizada foi o programa R, executado através do software Rstudio. Como ferramenta gráfica, foi empregado o

Quadro 1. Classificação dos deslizamentos

\begin{tabular}{|c|c|c|}
\hline Código & Classificação & Tipo de evento \\
\hline $\mathrm{E}$ & Escorregamento & $\begin{array}{l}\text { Agrupa as terminologias relativas ao movimento do solo utilizadas pelos jor- } \\
\text { nais: queda de barreiras, deslizamento, desmoronamento e escorregamento }\end{array}$ \\
\hline QB & Queda de blocos & $\begin{array}{l}\text { Casos relacionados ao desprendimento de blocos e de lajes de rocha com a } \\
\text { movimentação por queda livre }\end{array}$ \\
\hline RB & Rolamento de blocos & Casos relacionados a rolamentos de matacões e blocos de rocha \\
\hline $\mathrm{CL}$ & Corrida de lama & $\begin{array}{l}\text { Processos de mobilização de material envolvendo solo e rocha, com muita } \\
\text { água, desenvolvendo-se ao longo de drenagens }\end{array}$ \\
\hline$D$ & Diversos & $\begin{array}{l}\text { Casos em que faltava clareza na informação. Ex.: desabamento, podendo } \\
\text { estar relacionado a escorregamentos ou a desabamentos de uma edificação, } \\
\text { solapamento etc. }\end{array}$ \\
\hline
\end{tabular}

Fonte: Adaptado de banco de dados do Instituto de Pesquisas Tecnológicas, São Paulo. 
programa de geoprocessamento espacial QGis. Ambos os softwares são gratuitos. Através do programa R, onde foi realizado o tratamento de dados, foram gerados resultados relativos às ocorrências de eventos e acumulados de chuva em análises temporais, usando como métricas estatísticas as correlações e as médias, por exemplo. Em relação ao programa QGis, foram gerados produtos de análises espaciais com base nos pontos de ocorrências dos eventos no município.

Em razão das diferenças metodológicas dos institutos citados, o número total de casos relevantes, após o tratamento dos dados e retirada de dados espúrios, se mostrou discrepante: por exemplo, os dados contabilizados pelo IPT apontaram maior número de casos quando comparados aos contabilizados pelo GEOCART, que não levou em consideração relatos pessoais e notícias, por exemplo.

Foram feitas correlações utilizando informações disponibilizadas pelo site da Prefeitura do Município de Petrópolis referentes aos mapas e à cartografia, com base nos dados do Sistema de Informação Geográfica (SIG), no qual foram obtidos mapas com informações de hidrografia, densidade populacional, ocupação urbana e rural da região, entre outros. Entre os dados de maior relevância estão aqueles obtidos no Shuttle Radar Topography Mission (SRTM) para o ano 2000, como os relativos à altitude, relevo e declividade, que foram georreferenciados com os registros de deslizamento no município, com a finalidade de estabelecer uma relação espacial entre os eventos.

\section{RESULTADOS E DISCUSSÕES}

Nas Figuras 2a e 2b, encontram-se os histogramas com o número total de ocorrências de deslizamentos por década. Em ambos os períodos se observa uma tendência de aumento no número de casos. A maioria dos casos registrados, de acordo com a contagem de ambos os institutos, foram classificados como escorregamentos. Ainda que os dados referentes aos casos de escorregamento e os casos não classificados fossem extraídos da análise, uma tendência de aumento no número de ocorrências de deslizamentos seria observada com o decorrer das décadas, mesmo que tenha ocorrido um decréscimo nestes registros, como na década de 1950 (Figura 2a). Esta tendência está presente também nas informações do GEOCART, embora sua série de dados não seja tão extensa quanto à do IPT. Foi observado que em seis anos, entre 2010 e 2015 , registrou-se cerca de $80 \%$ do total de casos observados na década anterior, de 2000 a 2009 (Figura 2b).

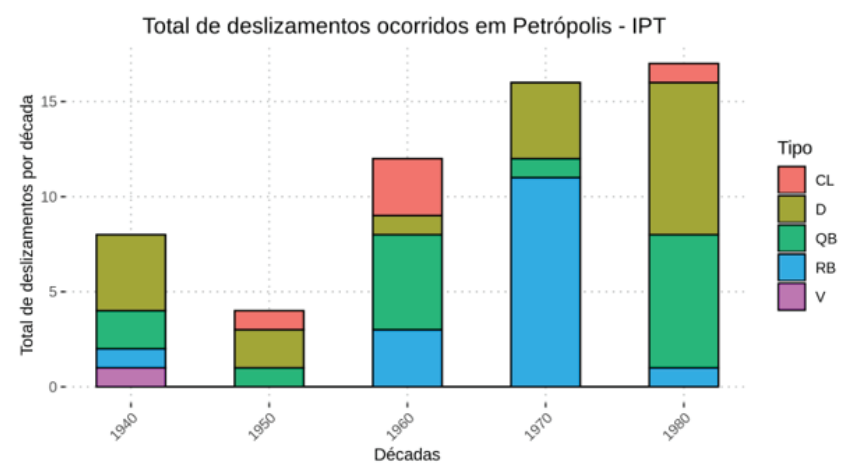

Figura 2a. Total de deslizamentos por década referente ao IPT entre 1940 e 1990, excluindo os casos de escorregamento e os não classificados.

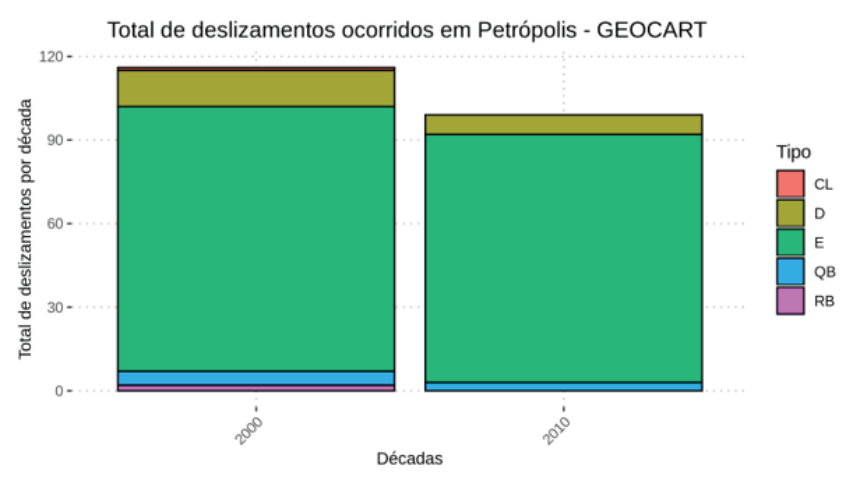

Figura $\mathbf{2 b}$. Total de deslizamentos por década referentes ao GEOCART entre 2000 e 2010.

Essa tendência encontrada nas Figuras $2 a$ e $2 b$ também foi observada de forma bem expressiva na Figura 3a, que indica a densidade dos deslizamentos nos períodos em análise segundo o IPT. Na Figura 3b, em relação ao GEOCART, os registros de eventos contabilizados mostraram um pico positivo no total de eventos no ano de 2011 e um decréscimo nos anos de 2014 e 2015, o que se pode atribuir à ocorrência de eventos meteorológicos nos referidos anos.

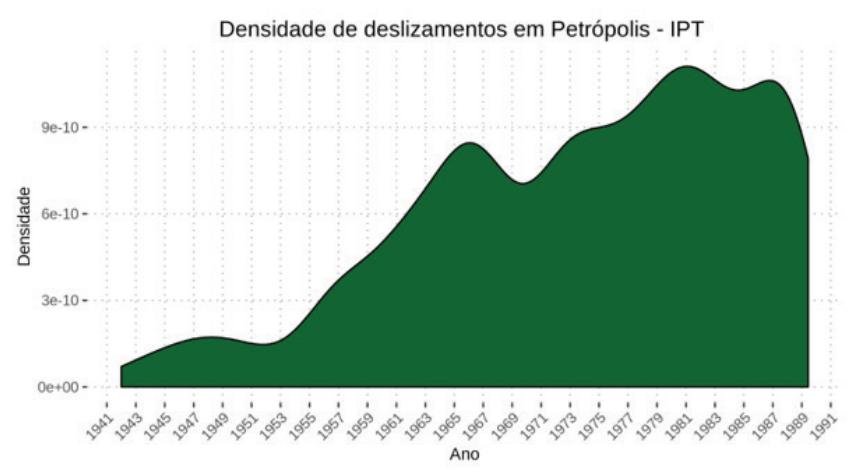




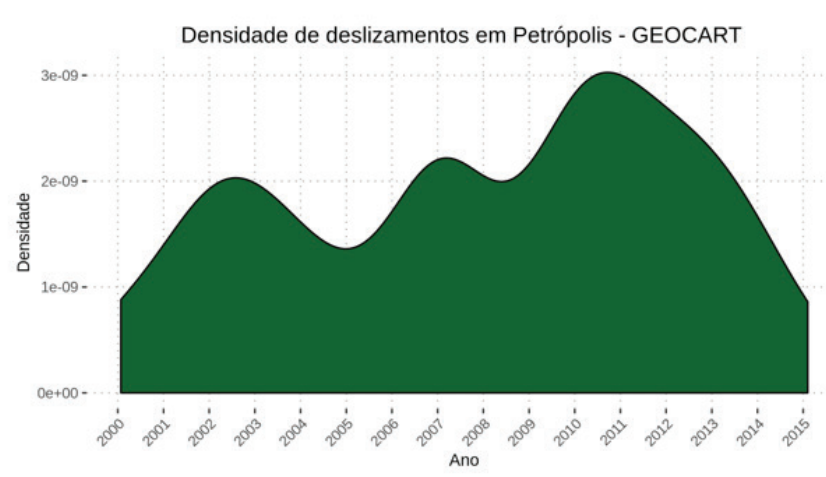

Figura 3a e Figura 3b. Densidade dos deslizamentos contabilizados pelo IPT (esquerda) e GEOCART (direita).

Nas Figuras 4a, 4b, 4c e 4d, encontram-se as distribuições temporais dos acumulados de precipitações de $24 \mathrm{~h}, 48 \mathrm{~h}$, $72 \mathrm{~h}$ e $96 \mathrm{~h}$, respectivamente, em relação ao tipo de evento e número de vítimas fatais. Os maiores registros foram os casos de escorregamento, os quais não apresentaram relação com a precipitação, assim como aqueles eventos classificados como diversos, em que não seria possível estabelecer correspondência. Por isso, estes dados puderam ser ocultados nesta correlação, além de serem desconside- rados nestes resultados. Juntamente com esses resultados, para cada evento foi considerada a contagem de vítimas, com o intuito de traçar um perfil do potencial destrutivo dos tipos de deslizamento tratados. Foi observada uma maior correspondência entre os eventos de Corrida de Lama $(C L)$, com maiores valores de precipitação e contagem de vítimas, principalmente no que se refere aos acumulados de $48 \mathrm{~h}$ e $72 \mathrm{~h}$.

A diferença entre o volume de dados dos institutos se mostrou um critério importante a ser considerado na abordagem dos dados de cada instituto, fazendo com que fosse necessária a adaptação dos resultados para o volume e tipo de dados obtidos. Com isso, nos dados referentes ao GEOCART, a correlação entre os eventos e os registros de precipitação não se mostraram satisfatórias.

Utilizando ainda os mesmos dados referentes ao IPT, nas Figuras $5 a, 5 b, 5 c$ e $5 d$, encontram-se os boxplots para os acumulados de $24 \mathrm{~h}, 48 \mathrm{~h}, 72 \mathrm{~h}$ e $96 \mathrm{~h}$, respectivamente. Pode-se notar uma menor dispersão de dados no evento $\mathrm{CL}$ quando relacionado aos acumulados de precipitação, principalmente no acumulado de $72 \mathrm{~h}$, mesmo considerando pontos outliers.

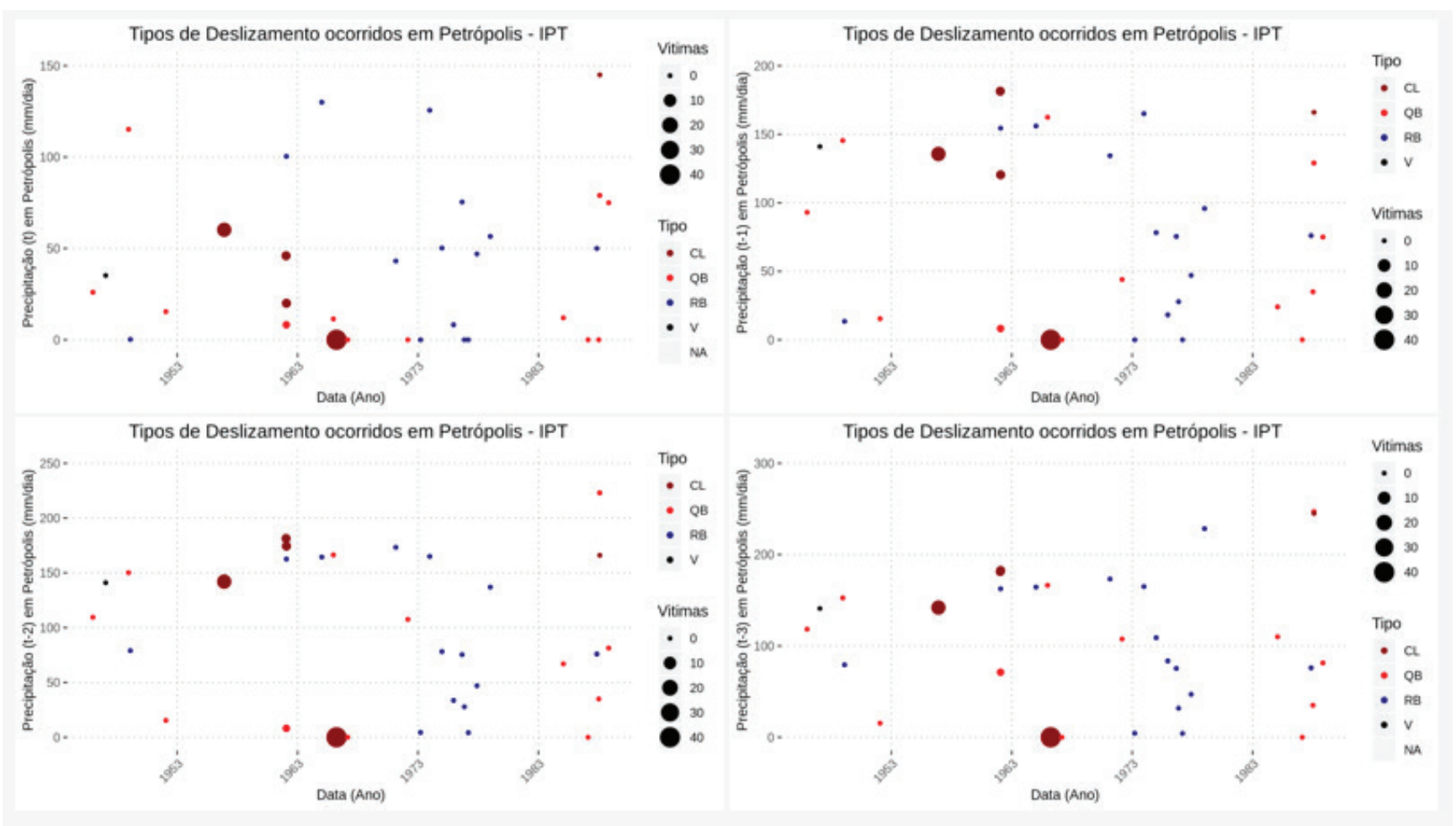

Figuras 4a (superior à direita). Correlação entre os tipos de deslizamento e o número de vítimas com o acumulado de chuva de $24 \mathrm{~h}$ em uma série temporal referentes ao IPT. Figura $\mathbf{4 b}$ (superior à esquerda). Correlação entre os tipos de deslizamento e o número de vítimas com o acumulado de chuva de $48 \mathrm{~h}$ em uma série temporal referentes ao IPT. Figura 4c (inferior à direita). Correlação entre os tipos de deslizamento e o número de vítimas com o acumulado de chuva de $72 \mathrm{~h}$ em uma série temporal referentes ao IPT. Figura $4 \mathrm{~d}$ (inferior à esquerda). Correlação entre os tipos de deslizamento e o número de vítimas com o acumulado de chuva de $96 \mathrm{~h}$ em uma série temporal 
Boxplot da precipitaçăo pelo tipo de Deslizamento - IPT

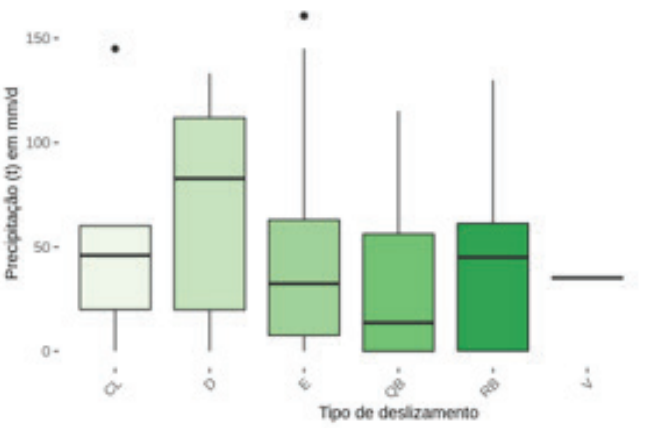

Boxplot da precipitação pelo tipo de Deslizamento - IPT

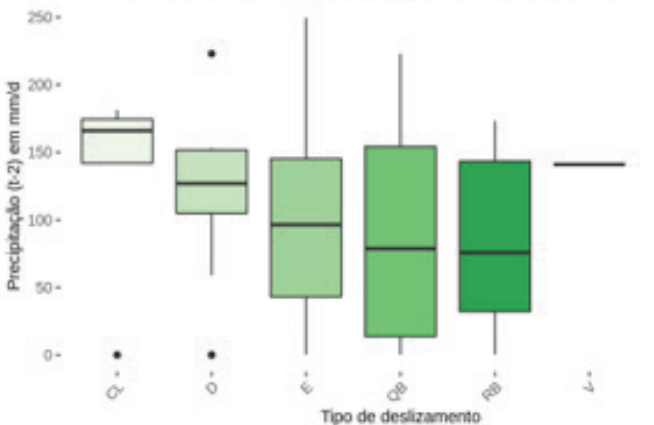

Boxplot da precipitação pelo tipo de Deslizamento - IPT
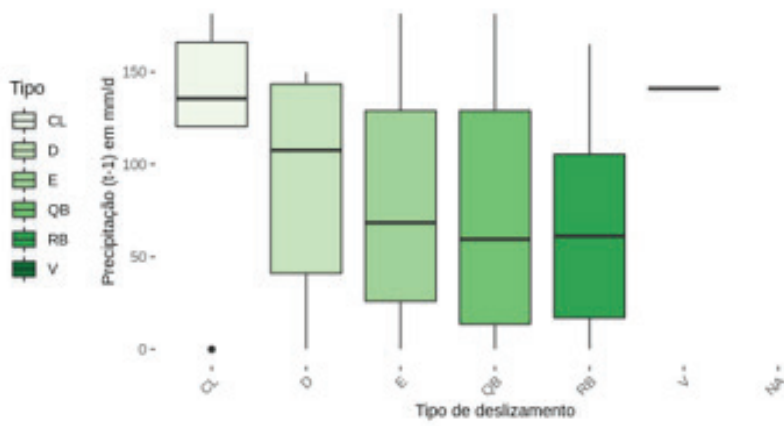

Boxplot da precipitação pelo tipo de Deslizamento - IPT

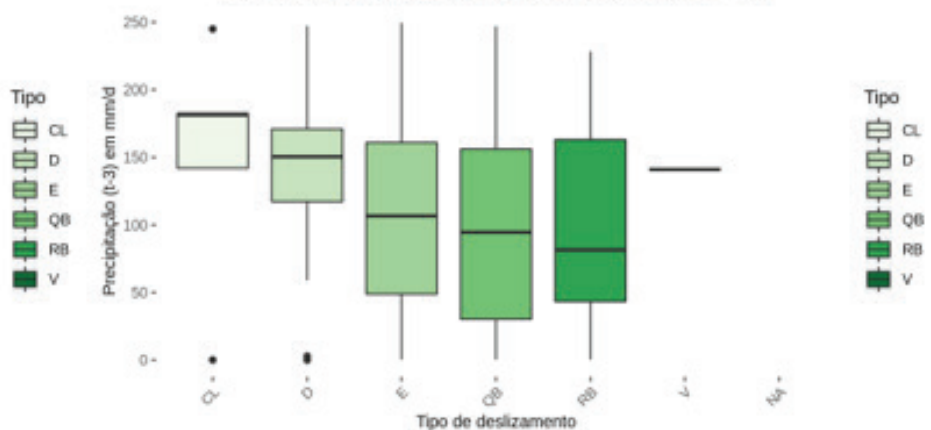

Figuras 5a (superior à direita). Boxplot dos tipos de deslizamento pelo acumulado de chuva em 24h referentes ao IPT. Figura 5b

(superior à esquerda). Boxplot dos tipos de deslizamento pelo acumulado de chuva em 48 h referentes ao IPT. Figura $5 \mathrm{c}$ (inferior à direita). Boxplot dos tipos de deslizamento pelo acumulado de chuva em $72 \mathrm{~h}$ referentes ao IPT. Figura $\mathbf{5} \mathbf{d}$ (inferior à esquerda). Boxplot dos tipos de deslizamento pelo acumulado de chuva em $96 \mathrm{~h}$ referentes ao IPT.

Precipitaçăo em Petrópolis (Funçăo Smooth) - IPT

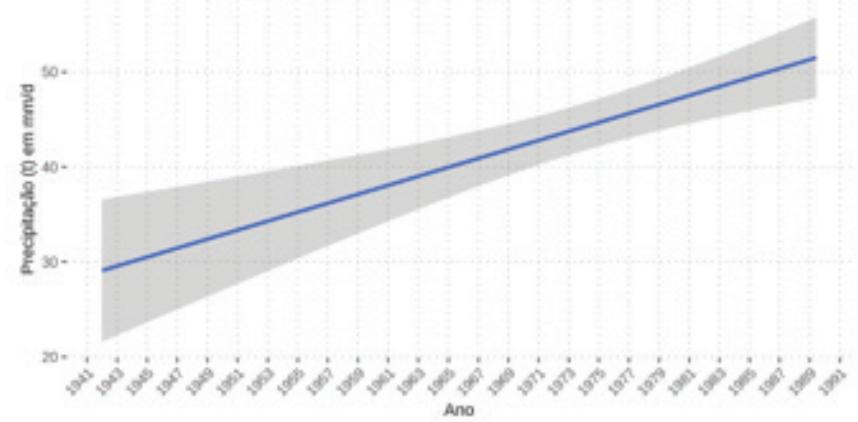

Precipitaçăo em Petrópolis (Funçẫo Smooth) - IPT

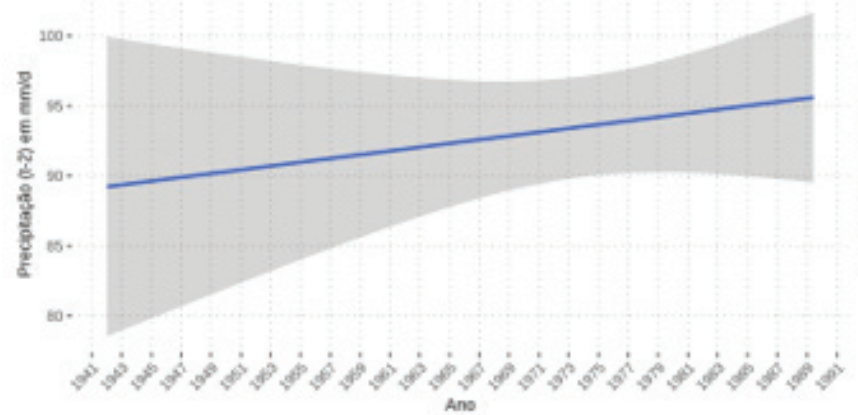

Precipitaçào em Petrópolis (Função Smooth) - IPT

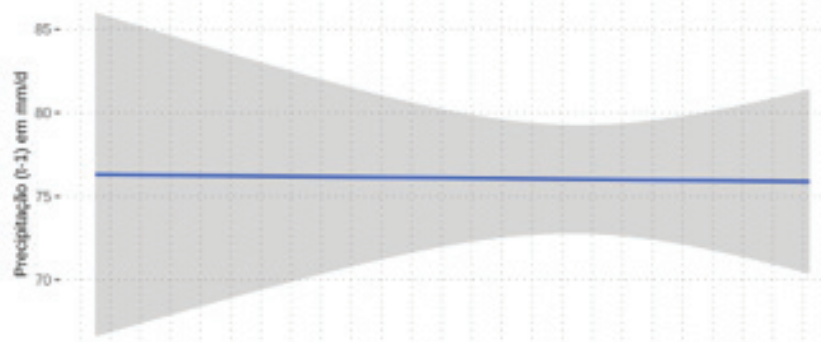

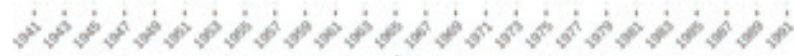

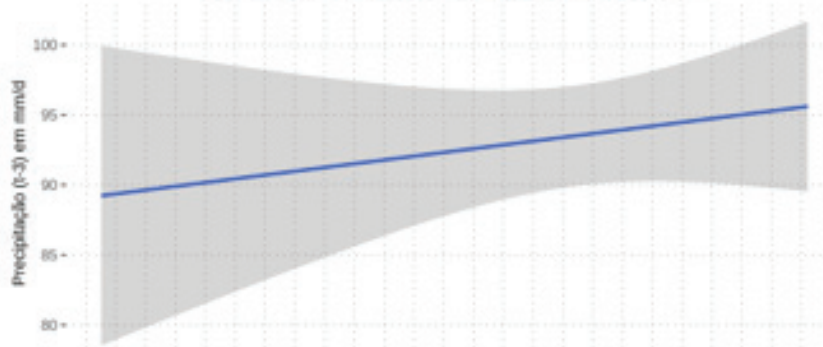

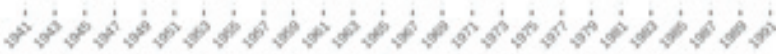

Ano

Figuras 6a (superior à direita). Função smooth da tendência de acumulado de precipitação em 24h em série temporal referente ao IPT.

Figura 6 b (superior à esquerda). Função smooth da tendência de acumulado de precipitação em $48 \mathrm{~h}$ em série temporal referente ao IPT. Figura $6 c$ (inferior à direita). Função smooth da tendência de acumulado de precipitação em $24 \mathrm{~h}$ em série temporal referente ao IPT. Figura $6 \mathrm{~d}$ (inferior à esquerda). Função smooth da tendência de acumulado de precipitação em 24h em série temporal referente ao IPT. 
Nas Figuras 6a, 6b, 6c e 6d estão apresentadas, respectivamente, as relações estabelecidas para os acumulados de precipitação de $24 \mathrm{~h}, 48 \mathrm{~h}, 72 \mathrm{~h}$ e $96 \mathrm{~h}$ (do período de 1940 a 1990), função Smooth. Essa função mostrou, de forma suavizada, uma tendência de aumento do acumulado de precipitação na estação em relação ao acumulado de $24 \mathrm{~h}$ (Figura $6 a)$, com pequena dispersão entre os dados. Em relação à tendência em $48 \mathrm{~h}$ (Figura 6b), foi observada estabilidade entre os acumulados, porém com maior dispersão entre os dados, principalmente no início da série temporal. Nas tendências de $72 \mathrm{~h}$ e $96 \mathrm{~h}$ (Figura 6c e Figura 6d) foram observados comportamentos semelhantes, com elevação menos acentuada e maior dispersão no início da série temporal.

Nas Figuras 7 e 8, com os dados obtidos com o GEOCART do período compreendido entre 2000 e 2015, encontram-se, respectivamente, a altitude, relevo e declividade da região (obtidos a partir do produto SRTM) e a distribuição espacial das ocorrências dos deslizamentos. Com base em dados de ocupação urbana, foi possível estabelecer uma relação entre os eventos dos deslizamentos com o relevo e o uso do solo. Os casos de deslizamentos estão relacionados a movimentos de descida de materiais em regiões em declive, sob o efeito da gravidade. Consequentemente, regiões de menor altitude, áreas mais baixas próximas a esses pontos de maior declive, são mais atingidas por estes eventos e enfrentam maiores consequências. Contudo, esta área de estudo ainda pode ser melhor explorada e desenvolvida por ser pertinente e essencial em diversos setores.

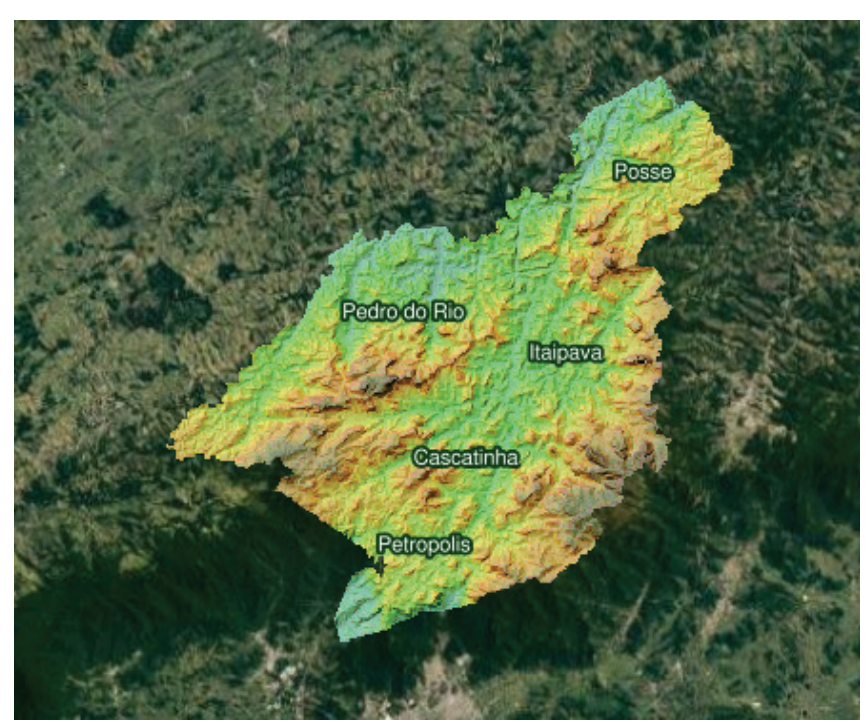

Figura 7. Produto SRTM com dados de altitude, relevo e declividade da região, 2000.

Fonte: Prefeitura de Petrópolis e Google.

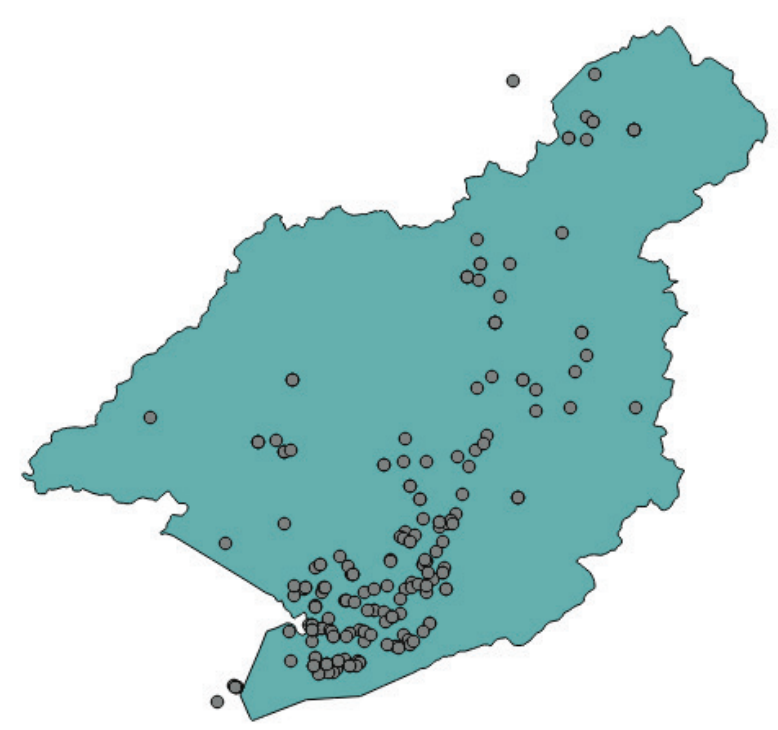

Figura 8. Representação espacial dos pontos de deslizamentos referentes ao GEOCART no período compreendido entre 2000 e 2015.

\section{CONCLUSÕES}

Este trabalho evidenciou a estreita relação entre os estudos de solo e atmosfera. Aliar estas temáticas é benéfico, quando se trata de gestão. Da mesma forma, seria possível integrar outras áreas de conhecimento de modo a otimizar processos e conhecer melhor as causas, impactos e consequências de determinado fenômeno.

Os resultados observados mostram a tendência de aumento dos eventos dos deslizamentos com o decorrer dos anos, assim como mostra certa tendência de aumento dos acumulados das precipitações, principalmente referentes a 24 horas. Essa tendência de aumento das ocorrências dos eventos mostra que há uma real necessidade de conhecer os tipos de evento, suas causas e danos, para com isso tentar prever a ocorrência desses fenômenos de forma que iniciativas possam ser tomadas a curto prazo para minimizar as consequências. Em casos de $\mathrm{CL}$, por exemplo, em que há uma maior relação entre os acumulados de chuva e contagem de vítimas, os acumulados de $72 \mathrm{~h}$ ( 3 dias antes do evento) mostraram maior associação com a ocorrência dos eventos, assim como também teve alguma relação com os acumulados de $24 \mathrm{~h}, 48 \mathrm{~h}$ e $96 \mathrm{~h}$. Fatores como estes, associados a um estudo da região tratada, podem ser levados em consideração no momento de traçar planos de prevenção dos eventos a fim de reduzir o perigo e consequentemente os danos que podem ser causados.

Observa-se que eventos meteorológicos estão relacionados ao maior número de ocorrências de deslizamentos, principalmente na Região Serrana, que possuem determinadas 
característica de relevo e declividade. Somando estes fatores com o uso do solo na região, é possível ter como resultado os locais mais vulneráveis em casos de deslizamentos e desta forma determinar melhores estratégias junto aos órgãos responsáveis. De acordo com a Figura 8, na região com maior ocupação urbana, 1을istrito de Petrópolis, o número de eventos dos deslizamentos foi maior em relação aos demais distritos. Essa mesma região, como visto na Figura 7, encontra-se entre pontos de maior relevo e altitude.

Por fim, a integração de estudos provenientes de diferentes áreas é aliada da gestão de riscos e desastres. Além disso, o conhecimento nestas áreas pode ser aplicado em diversas ocorrências. O Município de Petrópolis é comumente atingido por eventos extremos (deslizamentos e transbordamentos) e os órgãos da prefeitura, como a Defesa Civil, por exemplo, precisam agir em conjunto com profissionais técnicos a fim de conhecer melhor as particularidades da região e os eventos que a atingem.

\section{AGRADECIMENTOS}

Os autores agradecem ao Laboratório de Cartografia (GEOCART), do Departamento de Geografia, localizado no Instituto de Geociências da Universidade Federal do Rio de Janeiro, por ter disponibilizado os dados que tornaram possível a realização deste trabalho.

\section{REFERÊNCIAS}

Carmo, L.F.R., Nascimento, M.M.S., Martins, C.A., Silva, L.F.C., Sousa, R.C., Mota, L.L.O., Cruz, A.L.S.C. 2011. Desenvolvimento de modelo estatístico de elevação do Rio Quitandinha. Revista INEANA 6, 72-88. http://www.inea.rj.gov.br/wp-content/uploads/2018/12/Revista-Ineana-6.3.pdf

Coelho Netto A.L., Sato, A.M., Avelar, A.S., Vianna, L.G.G., Araujo, I.S., Croix, D., Lima, P., Silva, A.P., Pereira, R. 2013. January 2011: The extreme landslide disaster in Brazil. In: Margottini, C., Canuti, P., Sassa, K. (eds). Landslide Science and Practice. Berlin, Heidelberg: Springer. https://doi.org/10.1007/978-3642-31319-6_51
D'Orsi, R.N. 2011. Correlação entre pluviometria e escorregamentos no trecho da Serra dos órgãos da rodovia federal BR-116 RJ (Rio - Teresópolis). Tese de doutorado, COPPE, Universidade Federal do Rio de Janeiro.

Guerra, A.J.T, Lopes, P.B.M, Santos Filho, R. D. 2007. Características geográficas e geomorfológicas da APA Petrópolis, RJ. Revista Brasileira de Geomorfologia 8, 77-86. http://www.Isie. unb.br/rbg/index.php/rbg/article/view/87/80

Highland, L.M., Bobrowsky, P. 2008. O Manual de Deslizamento: Um Guia para a Compreensão de Deslizamentos. Reston, Virginia: U.S. Geological Survey.

Instituto Brasileiro de Geografia e Estatística (IBGE). 2020. IBGE Cidades. Petrópolis. https://cidades.ibge.gov.br/brasil/ $\mathrm{rj} /$ petropolis/panorama

Instituto Chico Mendes de Conservação da Biodiversidade (ICMBio). 2020. APA da Região Serrana de Petrópolis. http:// www.icmbio.gov.br/portal/unidadesdeconservacao/biomas-brasileiros/mata-atlantica/unidades-de-conservacao-mata-atlantica/2178-apa-de-petropolis

Instituto Estadual do Ambiente (INEA). 2020. Comitês de Bacias Hidrográficas - CBHs. http://www.inea.rj.gov.br/Portal/ Agendas/GESTAODEAGUAS/RECURSOSHIDRICOS/Comitedebacias/index.htm\&lang=PT-BR

Instituto Estadual do Ambiente. 2013. Resolução CERHI-RJ no107, de 22 de maio de 2013. Estado do Rio de Janeiro, Diário Oficial. https://www.comiteguandu.org.br/legislacoes/ResolucoesCERHI/Resolucao-CERHI-107.pdf

Licco, E.A. 2013. Vulnerabilidade social e desastres naturais: uma análise preliminar sobre Petrópolis, Rio de Janeiro. Revista de Saúde, Meio Ambiente e Sustentabilidade 8, 25-41. http://www3.sp.senac.br/hotsites/blogs/InterfacEHS/wp-content/uploads/2013/07/2_DOSSIE_vol-8n1.pdf

Prefeitura Municipal de Petrópolis. http://sig.petropolis. rj.gov.br/lm/index.php/view/map/?repository=publico\&proj ect=petropolis

Salles, R.O., Amaral, C. 2013. Estudo da correlação entre chuvas e escorregamentos na Região Serrana do Rio de Janeiro. Conferência Brasileira de Encostas, 6, Angra dos Reis.

Recebido: 10 fev. 2020

Aprovado: 06 mar. 2020

DOI: 10.20985/1980-5160.2020.v15n1.1611

Como citar: Torres, G.P., Carmo, L.F.R., Palmeira, A.C.P.A. (2020), Estudo da relação entre precipitação e deslizamentos no município de Petrópolis - RJ, Revista S\&G 15, No. 1, 38-45. https://revistasg.emnuvens.com.br/ sg/article/view/1611 